\title{
Retraction: Oligoarginine peptides slow strand annealing and assist non-enzymatic RNA replication
}

\author{
Tony Z. Jia, Albert C. Fahrenbach, Neha P. Kamat, Katarzyna P. Adamala and Jack W. Szostak
}

Nature Chemistry 8, 915-921 (2016); published online 27 June 2016; retracted after print 12 October 2017.

We the authors are retracting this Article because our efforts to repeat and follow up on the results have been unsuccessful. Specifically, we have been unable to reproduce observations suggesting that arginine-rich peptides allow the non-enzymatic copying of an RNA template in the presence of its complementary strand (Fig. 4e). We originally dismissed variability in these experiments as resulting from variability in the snap cooling of samples following thermal denaturation. However, we now understand that the data reported in the published article are the result of false positives that arose from an incorrectly designed experiment in which random errors, including transfer and concentration errors, affected the ratio of the concentrations of the RNA template and its complementary strand. This resulted in false positives that were misinterpreted as template copying in the presence of a complementary strand, where in reality these reactions did not contain enough complementary strands to completely inhibit the reaction.

Subsequent experiments suggested that arginine-rich peptides may not slow the reannealing of complementary strands (Fig. 3), and that what we had previously interpreted as a decrease in annealing rate was actually an artefact due to slow coalescence or strand exchange between droplets of RNA-peptide coacervate, as well as droplet coalescence and settling that led to decreased fluorescence intensity. Similarly, the changing circular dichroism spectra shown in Figure 2c, which were originally interpreted to be the result of a change in the global helical structure of RNA upon peptide binding, may also be an artefact due to, for example, loss of signal or light scattering. Although the binding of arginine-rich peptides to RNA does form condensed-phase droplets, and although most of the RNA does reside within the condensed phase, follow-up experiments to confirm that non-enzymatic RNA polymerization occurs within these coacervate droplets have been inconclusive (Fig. 5d).

The experiments showing that vesicles are stable in the presence of arginine-rich peptides (Supplementary Figure 26, by N. Kamat), and the failure of acidic peptides to condense RNA (Supplementary Figure 8, by K. Adamala) have been reproduced. However, since the main conclusions of our paper are incorrect, all of the authors are now retracting the Article. The authors would like to thank Dr Tivoli Olsen for her extensive efforts to unravel the errors in our Article and we apologize to the scientific community for any confusion arising from our publication. 